\section{SAT0203 OPTIMIZATION OF BIOLOGIC TAPERING USING ULTRASOUND IN RA PATIENTS}

S. Bawa ${ }^{1}$, M. Todd' $^{1}$, J. France ${ }^{1} .{ }^{1}$ Dept of Rheumatology, Gartnavel General Hospital, Glasgow, United Kingdom

Background: Optimal treatment using Treat 2 Target regimen has reduced morbidity and mortality rates in RA patients. However the use of biological therapies is expensive and a huge financial burden on Health budgets.

Current guidelines suggest to consider tapering biological therapies in patients with sustained low disease activity ${ }^{1}$.

There is a risk of overtreatment in this cohort, with potential risks from sustained immunosuppression of increased infection rates and the chance of malignancy.

Objectives: Studies have shown biologic tapering is possible. Some studies have performed progressive tapering using DAS28 scores, others with ultrasonography assessment ${ }^{2}$.

We used a progressive tapering strategy in tapering biological DMARDS in a selected RA cohort in a busy UK University Teaching Hospital, using ultrasound to guide the tapering process throughout and for detecting early recurrence during longer term followup.

Methods: Inclusion Criteria: Patients identified from routine clinic appointments as being either in clinical remission (DAS 28<2.6), or with low disease activity (DAS $28<3.2$ and no swollen joints), and with no flares of their RA for at least 12 months.

Assessment: At each clinic visit the patients' joints were examined, a DAS 28 and $\mathrm{HAQ}$ completed. Ultrasound was performed on hand and wrist joints (MCPJ's, PIPJ's, Wrists) in both Grey scale and Power Doppler to assess for inflammation. Biologic medication was progressively tapered according to results. Adalimumab was tapered to ' 3 weekly - 4 weekly - stop' and Etanercept to '2 weekly -3 weekly - stop'. Patients were given 3 monthly appointments. If patient flared or Ultrasound showed active synovitis, tapering was stopped and medication adjusted according to the findings. Pateints were followed up for a year at 3 monthly intervals, a year at 6 monthly intervals and then referred back to routine outpatient clinic.

Results: 28 patients were identified on Adalimumab and 8 on Etanercept.

Adalimumab: 17 patients $(61 \%)$ stopped completely. At the time of writing, 16 $(94 \%)$ remained off at 6 months. $12(71 \%)$ at 12 months and $5(29 \%)$ for $>23$ months. $6 / 17$ flared and had to restart medication -3 returned to a 4 weekly dose, 1 to 3 weekly and 2 to 2 weekly. $11 / 17(65 \%)$ remain off medication.

At present 7 patients (25\%) are on a 4 weekly dose, $1(4 \%)$ patient is on a 3 weekly dose and $9(32 \%)$ remain on 2 weekly.

Etanercept: 4 patients (50\%) stopped completely. 1 of these has remained off for over 12 months and 1 over 24 months. 1 of these patients returned to weekly injections within 4 months

1 patient (12.5\%) is on a 2 weekly dose and $4(50 \%)$ remain on weekly

The management of $72 \%$ of patients was optimised using Ultrasonography. Ongoing cost savings as a result of tapering were in the region of $£ 250,000$.

Conclusions: Our study shows Ultrasound significantly aided in successful biologic tapering. It helped in selection of appropriate patients, as well as in monitoring during/after tapering. It also resulted in significant cost savings in the region of $£ 250,000$

\section{REFERENCES:}

[1] Smolen JS, et al. EULAR recommendations for the management of rheumatoid arthritis with synthetic and biological disease-modifying antirheumatic drugs: 2013 update. Ann Rheum Dis 73(3):492-509.

[2] Alivernini S, et al. Tapering and discontinuation of TNF-alpha blockers without disease relapse using ultrasonography as a tool to identify patients with rheumatoid arthritis in clinical and histological remission. Arthritis Res Ther 18:39.

Acknowledgements: Rheumatology Unit GGH

Disclosure of Interest: None declared

DOI: 10.1136/annrheumdis-2018-eular.5199
SAT0204

NAÏVE AND RECENT THYMIC EMIGRANT CD4+ T CELLS INCREASE IN RHEUMATOID PATIENTS TREATED WITH ABATACEPT.

S. Piantoni ${ }^{1}{ }^{12}$, F. Regola ${ }^{1}$, A. Tincani ${ }^{1}$, P. Airò ${ }^{1} .{ }^{1}$ Rheumatology and Clinical Immunology Unit, Spedali Civili and University of Brescia, ${ }^{2}$ Department of Molecular and Translational Medicine, University of Brescia, Brescia, Italy

Background: $C D 4+T$ cells in rheumatoid arthritis $(R A)$ display a peculiar restriction of the T-cell receptor (TCR) repertoire which compromises their ability to react to novel antigens (1). We demonstrated that this process could be partially reverted by abatacept (ABA), which is a blocker of T lymphocyte co-stimulation, used in the treatment of RA (2). This effect could be at least in part due to a reduced generation of oligoclonal effector T cells, such as CD4+CD28-T cells (2). To better understand the mechanisms underlying this phenomenon, we speculated that ABA could influence the frequency of other peripheral T cell subpopulations, even at early stages of maturation. The homeostasis of the naïve $T$ cell poo is maintained not only by thymus production, but also by mechanisms of peripheral replication involving TCR activation, which can be revealed by CD31 (PECAM-1) down-modulation (3). Indeed, CD4+CD45RA+CD31+ T cells have been described as recent thymic emigrants (RTE) newly produced by the thymus and CD4+CD45RA+CD31- T cells as central naïve, involved in a self-renewal peripheral process. This latter population may have a restriction of TCR repertoire and was shown to be involved in mechanisms of cardiovascular damage in patients with coronary syndrome (4).

Objectives: We aimed at profiling circulating naïve CD4+CD45RA+ $T$ cells, by assessing their expression of CD31.

Methods: Thirty-one RA patients (median age [10 $10^{\circ}-90^{\circ}$ percentile] 42 [25-64] years) were evaluated before and after 6 months of ABA therapy. The response to treatment was evaluated with the EULAR criteria. Phenotypic analysis of peripheral blood T lymphocytes was made by flow-cytometry.

Results: After ABA therapy, the absolute number of total T CD4+ increased from 780 [423-1351] to 1000 [658-1566] cells $/ \mathrm{mm} 3(\mathrm{p}=0.01)$. Total naïve CD4+ increased in percentage (33 [18-56] vs $40[20-61] \%$ of $C D 4+; p=0.02)$ and in absolute number (257 [82-568] vs 344 [62-689] cells/mm3; $p=0.03)$. In parallel, the number of RTE increased in percentage (10.6 [2-26] vs 11.6 [3-25] \% of CD4 $+; \mathrm{p}=0.04)$ and in absolute number $(51$ [15-194] vs 110 [23-271] cells $/ \mathrm{mm3}$; $\mathrm{p}=0.01)$. The central naïve counterpart did not show significant variations in percentage (29 [23-40] vs 27 [20-38] \% of $C D 4+; \mathrm{p}=0.20$ ) nor in absolute number (191 [41-404] vs 242 [76-526] cells/mm3; $p=0.20$ ). The modifications of T cell number were not significantly different when good and moderate responder $(n=22)$ and no responder $(n=9)$ patients were compared, at baseline and after therapy. No correlation was found between age of patients, clinical features of the disease and RTE number at baseline and after 6 months .

Conclusions: The number of total naïve $T$ cells increases after therapy with ABA together with the number of RTE, suggesting a thymic output boost. Besides the peripheral effect in reducing the number of effector $T$ cells which was showed by previous studies (5), ABA could have a role in promoting the immune reconstitution at the early stage of $\mathrm{T}$ cell development. Furthermore, a consequent favorable effect on possible cardiovascular damage mechanisms mediated by CD31T cells might be hypothesized.

\section{REFERENCES}

[1] Wagner UG, et al. PNAS 1998

[2] Imberti L, et al. J Transl Med 2015.

[3] Kohler S, et al. Blood 2009.

[4] Flego D, et al. Basic Res Cardiol 2014;109:448.

[5] Airò P, et al. Trends Mol Med 2013.

Acknowledgements: Bristol-Myers-Squibb Italy provided an unrestricted research grant.

Disclosure of Interest: None declared

DOI: 10.1136/annrheumdis-2018-eular.2534

\section{SAT0205 COMPARISON OF DISEASE STATUS IN UK PATIENTS WITH RA RECEIVING TNFI VS CDMARD}

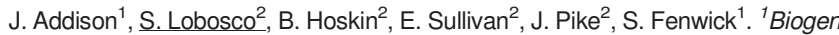
Idec, Maidenhead, ${ }^{2}$ Adelphi Real World, Bollington, United Kingdom

Background: UK NICE guidance recommends initiation of tumour necrosis factor-alpha inhibitor (TNFi) for patients with severe RA unresponsive to intensive 
therapy with conventional disease-modifying anti-rheumatic drugs (cDMARD). Data comparing CDMARD and TNFi in clinical trial settings are common, but there is limited published real-world evidence on their comparative effectiveness.

Objectives: Compare disease status and outcomes in UK patients with RA receiving cDMARD vs TNFi.

Methods: Data derive from Adelphi's RA Disease Specific Programme 2017, a cross-sectional survey of 49 UK rheumatologists providing information on demographics, disease history, disease status and treatment of patients with RA. All patients $(n=640)$ were included in the RA treatment analysis; patients were included in the comparative analysis if at the time of survey they had been receiving current treatment for at least 3 months, with either cDMARD (and had never received biologic(b)DMARD), or a TNFi (and had never received prior bDMARD). A propensity score based on BMI, duration on current therapy, RA severity and disease duration at initiation of current therapy was used to match treatment groups. Using Abadie-Imbens standard errors, clinical characteristics and measures of disease activity were compared between the matched groups.

Results: Current therapy of 640 patients: 379 (59.2\%) bDMARD, of which 253 (66.8\%) were receiving TNFi; 212 (33\%) cDMARD; $18(2.8 \%)$ had never received any DMARD, 15 (2.3\%) had discontinued bDMARD. Mean DAS28 at initiation of therapy was 4.94 in overall cDMARD and 5.77 in TNFi groups. Table 1 shows the comparative analysis of cDMARD vs TNFi matched treatment groups. The CDMARD group had a higher proportion of moderate/severe and active/very active disease at time of survey, a higher proportion of patients in this group had no improvement in disease severity or activity since initiation of current therapy, and they were less likely to have achieved a EULAR response. A higher proportion of CDMARD patients and physicians were not satisfied with their disease control. Mean DAS28 scores at time of survey had declined from time of initiation of therapy to time of survey in matched cDMARD vs TNFi groups, from 5.27 to 3.1 vs 5.77 to 2.7 respectively.

Table 1

\begin{tabular}{|c|c|c|c|}
\hline & $\%$ & $\%$ & $P=$ \\
\hline \multicolumn{4}{|l|}{ Physician reported current disease status } \\
\hline Moderate or severe & 43.5 & 22.4 & 0.02 \\
\hline 'Active' or 'very active' disease & 58.8 & 44.1 & 0.10 \\
\hline \multicolumn{4}{|l|}{$\begin{array}{l}\text { Change in disease status since initiation of current } \\
\text { treatment }\end{array}$} \\
\hline 'No improvement' in severity & 25.3 & 7.7 & 0.04 \\
\hline 'No improvement' in disease activity ${ }^{1}$ & 22.0 & 7.7 & 0.08 \\
\hline Not achieved EULAR response (based on DAS28) ${ }^{2}$ & 16.0 & 6.9 & 0.08 \\
\hline \multicolumn{4}{|l|}{ Satisfaction with current disease control } \\
\hline Physicians 'not satisfied' & 39.4 & 8.2 & $<0.01$ \\
\hline Patients 'not satisfied' 3 & 31.7 & 16.7 & 0.35 \\
\hline
\end{tabular}

Lower sample size due to missing values: ${ }^{1}$ cDMARD 147, TNFi $168 ;{ }^{2}$ cDMARD 75, TNFi 131; ${ }^{3}$ cDMARD 54, TNFi 60

Conclusions: UK patients with RA receiving cDMARD have poorer outcomes, in terms of measured disease status and control, than their matched counterparts receiving TNFi. Despite having a higher mean DAS score at initiation, patients in the TNFi group had a lower mean score at time of survey than patients receiving cDMARD. This real-world evidence highlights the continued utility of TNFi as effective treatments for patients with RA. Potential benefit of early TNFi initiation merits further research.

Disclosure of Interest: J. Addison Shareholder of: Biogen, Employee of: Biogen, S. Lobosco Employee of: Adelphi Real World, B. Hoskin Employee of: Adelphi Real World, E. Sullivan Employee of: Adelphi Real World, J. Pike Employee of: Adelphi Real World, S. Fenwick Shareholder of: Biogen, Employee of: Biogen DOI: 10.1136/annrheumdis-2018-eular.5812

\section{SAT0206 \\ RESULTS OF THE ALTERRA CLINICAL TRIAL - THE EFFICACY OF THE ALTERNATIVE DOSING REGIMEN FOR RITUXIMAB BIOSIMILAR IN BDMARDS NAIVE PATIENTS WITH RHEUMATOID ARTHRITIS}

V. Mazurov ${ }^{1}$, L. Denisov ${ }^{2}$, I. Gordeev ${ }^{3}$, O. Nesmeyanova ${ }^{4}$, T. Plaksina ${ }^{5}$, E. llivanova ${ }^{6}$, D. Krechikova ${ }^{7}$, E. Zonova ${ }^{8}$, L. Knyazeva ${ }^{9}$, A. Artemeva ${ }^{10}$, E. Dokukina ${ }^{10}, \mathrm{E}$. Chernyaeva ${ }^{10}, \mathrm{R}$. Ivanov ${ }^{10} .{ }^{1}$ North-Western State Medical University named after I.I. Mechnikov, Saint-Petersburg, ${ }^{2}$ V.A. Nasonova Research Institute of Rheumatology, ${ }^{3}$ O.M. Filatov City Clinical Hospital №15, Moscow, ${ }^{4}$ Chelyabinsk Regional Clinical Hospital, Chelyabinsk, ${ }^{5}$ N.A. Semashko Nizhniy Novgorod Regional Clinical Hospital, Nizhniy Novgorod, ${ }^{6}$ Leningrad Regional Clinical Hospital, Saint-Petersburg, ${ }^{7}$ The Department Hospital at Smolensk Station of the open JSC "Russian Railways", Smolensk, ${ }^{8}$ Novosibirsk State Medical University, Novosibirsk, ${ }^{9}$ Kursk Reginal Clinical Hospital, Kursk, ${ }^{10}$ JCS BIOCAD, Saint-Petersburg, Russian Federation

Background: Rituximab (RTX) is successfully used in patients with active rheumatoid arthritis (RA) who previously received biological disease-modifying antirheumatic drugs (bDMARDs) at a dose of $1000 \mathrm{mg}$. Previous preclinical and clinical studies showed that BCD-020 is highly similar to innovator RTX. ALTERRA study demonstrated that first-line use of $600 \mathrm{mg} \mathrm{BCD-020}$ is very effective in bDMARDs naive patients with RA

Objectives: The goal of ALTERRA study was to evaluate the efficacy and safety of the alternative dosing regimen $(600 \mathrm{mg})$ of BCD-020 in bDMARDs naive patients with RA

Methods: ALTERRA study was conducted as multicenter randomized doubleblind placebo-controlled phase 3 study. After the screening patients were stratified by age, anti-CCP level and DAS28 score, randomized (2:1) into 2 arms and received BCD-020 (in combination with methotrexate (MTX)) in a dose $600 \mathrm{mg}$ IV or placebo (in combination with MTX) on day 1 and day 15 , then, if the activity of arthritis persisted after 24 wks of study, a second course was provided. Patients were observed up to 52 wks.

Results: A total of 159 patients were enrolled in ALTERRA study, 107 patients in BCD-020 arm and 52 patients in placebo arm.

Efficacy: ACR20 at wk 24 was reached by $65.69 \%$ of patients in BCD-020 arm and $29.41 \%$ in placebo arm ( $p=0.00005$, the difference in proportion of registration ACR20 with $95 \% \mathrm{Cl}$ was 29.41 [19.27\%; 53.28\%], margin $10.5 \%$ ) in per protoco population, so hypothesis of superiority was confirmed. The performed analysis showed a much more pronounced decrease in the DAS28-4 (ESR) index in BCD 020 arm compared with placebo arm $(\mathrm{p}=0.0006)$ at wk 24. A much more significant decrease in change of the HAQ-DI index was also shown in the BCD-020 arm $(p=0.008)$. Analysis of efficacy at wk 52 showed the preservation of the response after 2 courses of therapy with BCD-020, $600 \mathrm{mg}$ (in combination with MTX): ACR20 reached by $84.5 \%$, ACR50 - by $54.6 \%$, ACR70 - by $29.9 \%$ of patients.

Safety: BCD-020 showed a favorable safety profile with no significant difference with placebo use (in combination with MTX). After 24 wks patients of both groups developed high similar level of related adverse events: $16.8 \%$ of patients in BCD 020 arm and $11.76 \%$ in placebo arm $(p=0.555)$. There were only 3 cases of severe adverse events (2.8\%) in BCD-020 arm and 2 cases (3.92\%) in placebo group From wk 24 to wk 52: $13.08 \%$ of patients (who received 2 courses of BCD-020) and $19.61 \%$ of patients (who received one course of BCD-020 after 24 wk) developed related adverse events.

Figure 1 ACR 20/50/70 in bDMARDs naive patients with RA after 24-wk treatment of BCD020/placebo (in combination with MTX)

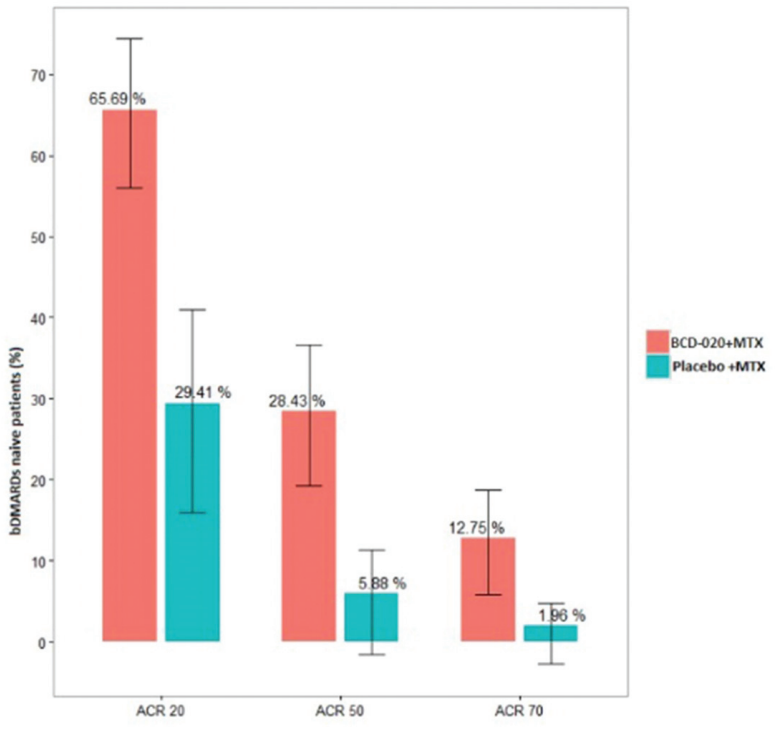

Conclusions: ALTERRA study showed high efficacy and favorable safety profile of RTX biosimilar BCD-020 at a dose of $600 \mathrm{mg}$ in combination with MTX in bDMARDs naive patients with RA

Disclosure of Interest: V. Mazurov: None declared, L. Denisov: None declared, I. Gordeev: None declared, O. Nesmeyanova: None declared, T. Plaksina: None declared, E. Ilivanova: None declared, D. Krechikova: None declared, E. Zonova: None declared, L. Knyazeva: None declared, A. Artemeva Employee of: JSC BIO CAD, E. Dokukina Employee of: JSC BIOCAD, E. Chernyaeva Employee of: JSC BIOCAD, R. Ivanov Employee of: JSC BIOCAD DOI: 10.1136/annrheumdis-2018-eular.2300 